\title{
UNITARIES AND PARTIAL ISOMETRIES IN A REAL $W^{*}$-ALGEBRA
}

\author{
KENNETH E. EKMAN
}

\begin{abstract}
The group of unitaries in a real $W^{*}$-algebra without a finite type I direct summand is connected. This fact is used to characterize the components of the set of partial isometries in such algebras.
\end{abstract}

1. Introduction. Many theorems for complex $W^{*}$-algebras apply equally well to real $W^{*}$-algebras, of ten with identical proofs, while others, such as the spectral theorem do not. However, we show in $\$ 2$ that one of the standard consequences of the spectral theorem, the connectivity of the unitary operators in a complex $W^{*}$-algebra, holds for real $W^{*}$-algebras without a finite type I summand. In $\S 3$ we use the result of $\S 2$ to extend to real $W^{*}$-algebras without a finite type I summand, and to all complex $W^{*}$-algebras, a result of Halmos and McLaughlin [4] characterizing the components of partial isometries.

For any real, separable, infinite-dimensional Hilbert space $\mathscr{H}$ let $\mathcal{H}_{c}=\mathscr{H}$ $\otimes \mathbf{C}$ be its complexification. We may identify $\mathcal{H}_{c}$ with $\mathcal{H} \oplus \mathcal{H}$ (as real spaces), and, if $A$ is a bounded (real) linear operator on $\mathcal{H}$, we may identify $A$ with the (complex) linear operator on $\mathcal{H}_{C}$ whose matrix on $\mathcal{H} \oplus \mathcal{H}$ is $\left(\begin{array}{ll}A & 0 \\ 0 & A\end{array}\right)$. Since multiplication by $i$ on $\mathscr{K}_{c}$ corresponds to the matrix $\left(\begin{array}{cc}0 & -I \\ I & 0\end{array}\right), B$ is a bounded (complex) linear operator on $\mathcal{H}_{c}$ if and only if $B$ is of the form $B_{1}+i B_{2}$, where $B_{1}$ and $B_{2}$ are bounded real linear operators on $\mathcal{H}$, i.e., matricially $B$ has the form

$$
\left(\begin{array}{cc}
B_{1} & -B_{2} \\
B_{2} & B_{1}
\end{array}\right) .
$$

If $\mathfrak{U}$ is a real $W^{*}$-algebra of operators on $\mathscr{H}$, let $\mathfrak{A}_{c}$ denote its complexification (that is, $\mathfrak{A}_{c}$ is just the set of all $A_{1}+i A_{2}$, where $A_{1}$ and $A_{2}$ are in $\mathfrak{U}$ ). With the canonical identifications above, $\mathfrak{A}_{c}$ is a complex $W^{*}$-algebra of operators on $\mathcal{H}_{c}$.

\section{The group of unitaries.}

THEOREM 1. The unitary group in a real $W^{*}$-algebra $\mathfrak{A}$ without a finite type I direct summand is arcwise connected.

Proof. Let $U$ be a unitary element of $\mathfrak{A}$. Then $U$ is also unitary in $\mathfrak{U}_{c}$ so we may use the spectral theorem to conclude $U=\exp (i H)$ for some Hermitian $H$ in $\mathfrak{A}_{c}$. Following Martin [6], we assume without loss of generality that $H$ is "reduced" (i.e., that $\sigma(H) \subset[0,2 \pi]$ and $2 \pi$ is not in the point spectrum of $H$ ).

Received by the editors May 29, 1974 and, in revised form, August 26, 1974.

AMS (MOS) subject classifications (1970). Primary 46L10. 
We may decompose $H$ into $\pi B+i S$, where $B$ is a selfadjoint element of $\mathscr{U}$ and $S$ is a skew-adjoint element of $\mathfrak{A}$. Since $U$ is actually in $\mathfrak{A}$ (hence, orthogonal), we may use a result of Martin [6, p. 602] to conclude that $B$ is idempotent and commutes with $S$. Thus, we have

$$
U=\exp (i H)=\exp (i \pi B-S)=\exp (i \pi B) \exp (-S) .
$$

Note that $B$ is actually a projection and that an easy calculation shows $\exp (i \pi B)=I-2 B$.

What we wish to do now is to find some skew-adjoint $T$ in $\mathscr{A}$ such that $\exp (\pi T)=I-2 B=\exp (i \pi B)$. Then if we set $U_{t}$ to be $\exp (t \pi T) \exp (-t S)$ for $0 \leqslant t \leqslant 1$, each $U_{t}$ lies in $\mathfrak{A}$ and is unitary in $\mathfrak{A}_{c}$, so we have constructed a continuous path of unitaries in $\mathfrak{A}$ joining $U$ to $I$. This, of course, will prove the theorem.

The operator $T$ is, roughly speaking, a square root of $-I$ on $B \mathcal{H}$ and zero on $(I-B) \mathcal{H}$. More precisely, we may apply Theorems 45 and 49 of Kaplansky [5] and the standard direct sum decomposition of $\mathfrak{A}$ to conclude that $B=Q_{1}+Q_{2}$, where $Q_{1}$ and $Q_{2}$ are equivalent orthogonal projections in $\mathscr{A}$. Thus, there is a partial isometry $V$ of $\mathfrak{A}$ with $V^{*} V=Q_{1}$, and $V V^{*}=Q_{2}$. We set $T$ to be $V-V^{*}$. Then $T$ is skew-adjoint, commutes with $B$, and satisfies $B T B=T$. Thus, if we consider $B$ and $T$ as elements of $\mathfrak{A}_{c}, T$ is an element of the $W^{*}$-algebra $B \mathfrak{A}_{c} B$. Then $R=-i T$ is a selfadjoint unitary in $B \mathfrak{A}_{c} B$, so its spectrum satisfies $\sigma(R) \subset\{+1,-1\}$. For $\lambda \neq 0,+1,-1$, let $C_{\lambda}$ be the inverse of $(R-\lambda)$ in $B \mathfrak{A}_{c} B$. Then $(I-B)\left(-\lambda^{-1}\right)(I-B)+B C_{\lambda} B$ is the inverse of $(R$ $-\lambda)$ in $\mathfrak{A}_{c}$, so the spectrum of $R$ in $\mathfrak{A}_{c}$ is contained in $\{0,+1,-1\}$. Let $E$ be the spectral measure on $\sigma(R)$ for the spectral decomposition of $R$. Then $E(\{+1\})$, $E(\{-1\})$, and $E(\{0\})$ are projections in $\mathfrak{A}_{c}$ such that

$$
E(\{+1\})+E(\{-1\})=B
$$

and, for a function $f$ on $\sigma(R)$,

$$
f(R)=f(+1) E(\{+1\})+f(-1) E(\{-1\})+f(0) E(\{0\}) .
$$

Setting $f(\lambda)=\exp (i \pi \lambda)$ yields

$$
\exp (i \pi R)=-E(\{+1\})-E(\{-1\})+E(\{0\})=-B+(I-B)=I-2 B .
$$

But $i \pi R$ is just $\pi T$, so $T$ is the operator for which we were searching. Q.E.D.

3. Partial isometries. If $E$ and $F$ are two projections of a $W^{*}$-algebra $\mathfrak{A}$, write $E \sim F$ if $E$ and $F$ are equivalent in the usual way, i.e., if there is a partial isometry $W$ of $\mathfrak{A}$ with $W^{*} W=E$ and $W W^{*}=F$. If $W$ and $V$ are any two partial isometries of $\mathfrak{U}$, let $E=W^{*} W, E^{\prime}=W W^{*}, F=V^{*} V$, and $F^{\prime}$ $=V V^{*}$. Write $W \approx V$ if $(I-E) \sim(I-F), E^{\prime} \sim F^{\prime},\left(I-E^{\prime}\right) \sim\left(I-F^{\prime}\right)$. (Note that this implies $E \sim F$ also, since $E \sim E^{\prime}$ and $F \sim F^{\prime}$.)

Lemma 1. Let $W$ and $V$ be partial isometries in a $W^{*}$-algebra $\mathfrak{A}$ (real or complex). If $\|W-V\|<\frac{1}{2}$, then $W \approx V$.

Proof. Using the notation of the previous paragraph, we have 


$$
\begin{aligned}
\|E-F\| & =\left\|W^{*} W-V^{*} V\right\| \leqslant\left\|W^{*}\right\|\|W-V\|+\left\|W^{*}-V^{*}\right\|\|V\| \\
& \leqslant 2\|W-V\|<1 .
\end{aligned}
$$

Similarly, $\left\|E^{\prime}-F^{\prime}\right\|<1$. The polar decomposition theorem holds for $\mathfrak{A}[5$, Theorem 65], so we may apply Lemma 1.2 of Araki, Smith, and Smith [1] to conclude $(I-E) \sim(I-F), E^{\prime} \sim F^{\prime}$, and $\left(I-E^{\prime}\right) \sim\left(I-F^{\prime}\right)$. Q.E.D.

In the opposite direction, we have the following result.

LEMMA 2. Let $\mathfrak{A}$ be a $W^{*}$-algebra in which the unitaries are arcwise connected. Let $W$ and $V$ be two partial isometries in $\mathfrak{A}$ such that $W \approx V$. Then there exists a continuous path $W_{t} \quad(0 \leqslant t \leqslant 1)$ of partial isometries of $\mathfrak{A}$ such that $W_{0}=W, W_{1}=V$, and $W_{t} \approx W$ for each $t$.

Note that this lemma extends the result of Halmos and McLaughlin [4] for the case that $\mathfrak{U}$ is the algebra of all bounded operators on a complex Hilbert space. In fact, the proof is practically identical to the proof (of Douglas) for that special case (see Halmos [3]) and will be omitted. (One need only note that the assumptions of the lemma suffice to insure that the partial isometries of Douglas' proof do lie in the $W^{*}$-algebra $\mathfrak{A}$.)

Combining Lemmas 1 and 2 we have the following result.

THEOREM 2. Let $\mathfrak{U}$ be a $W^{*}$-algebra whose unitary group is arcwise connected. Then two partial isometries $W$ and $V$ of $\mathfrak{A}$ are in the same component of the set of partial isometries if and only if $W \approx V$.

COROLLARY. If $W$ and $V$ are two partial isometries in a real $W^{*}$-algebra with no finite type I direct summand, then $W$ and $V$ are in the same component of the set of partial isometries if and only if $W \approx V$.

\section{REFERENCES}

1. H. Araki, M.-S. B. Smith and L. Smith, On the homotopical significance of the type of the von Neumann algebra factors, Comm. Math. Phys. 22 (1971), 71-88. MR 44 \#5783.

2. R. K. Goodrich, The spectral theorem for real Hilbert space, Acta Sci. Math. (Szeged) 33 (1972), 123-127. MR 46 \# 4253.

3. P. R. Halmos, A Hilbert space problem book, Van Nostrand, Princeton, N.J., 1967. MR 34 $\# 8178$.

4. P. R. Halmos and J. E. McLaughlin, Partial isometries, Pacific J. Math. 13 (1963), 585596. MR $28 \# 477$.

5. I. Kaplansky, Rings of operators, Benjamin, New York, 1968. MR 39 \#6092.

6. M. H. Martin, On infinite orthogonal matrices, Amer. J. Math. 54 (1932), 579-631.

7. C. R. Putnam and A. Wintner, The connectedness of the orthogonal group in Hilbert space, Proc. Nat. Acad. Sci. U.S.A. 37 (1951), 110-112. MR 13, 10.

8. - The orthogonal group in Hilbert space, Amer. J. Math. 74 (1954), 52-78. MR 13, 531.

Department of Mathematics, Duke University, Durham, North Carolina 27706

Current address: Center for Naval Analyses, 1401 Wilson Boulevard, Arlington, Virginia 22209 